\title{
Anaesthesia for neurosurgical procedures in pregnant patients: a systematic review
}

\author{
Lauretta MP, Lubnin A, Fomberstein KM, Bosellino B, Spennati V, Bilotta F.
}

Algorithm for management of brain tumors in pregnant women (modified from Tewari et al ).
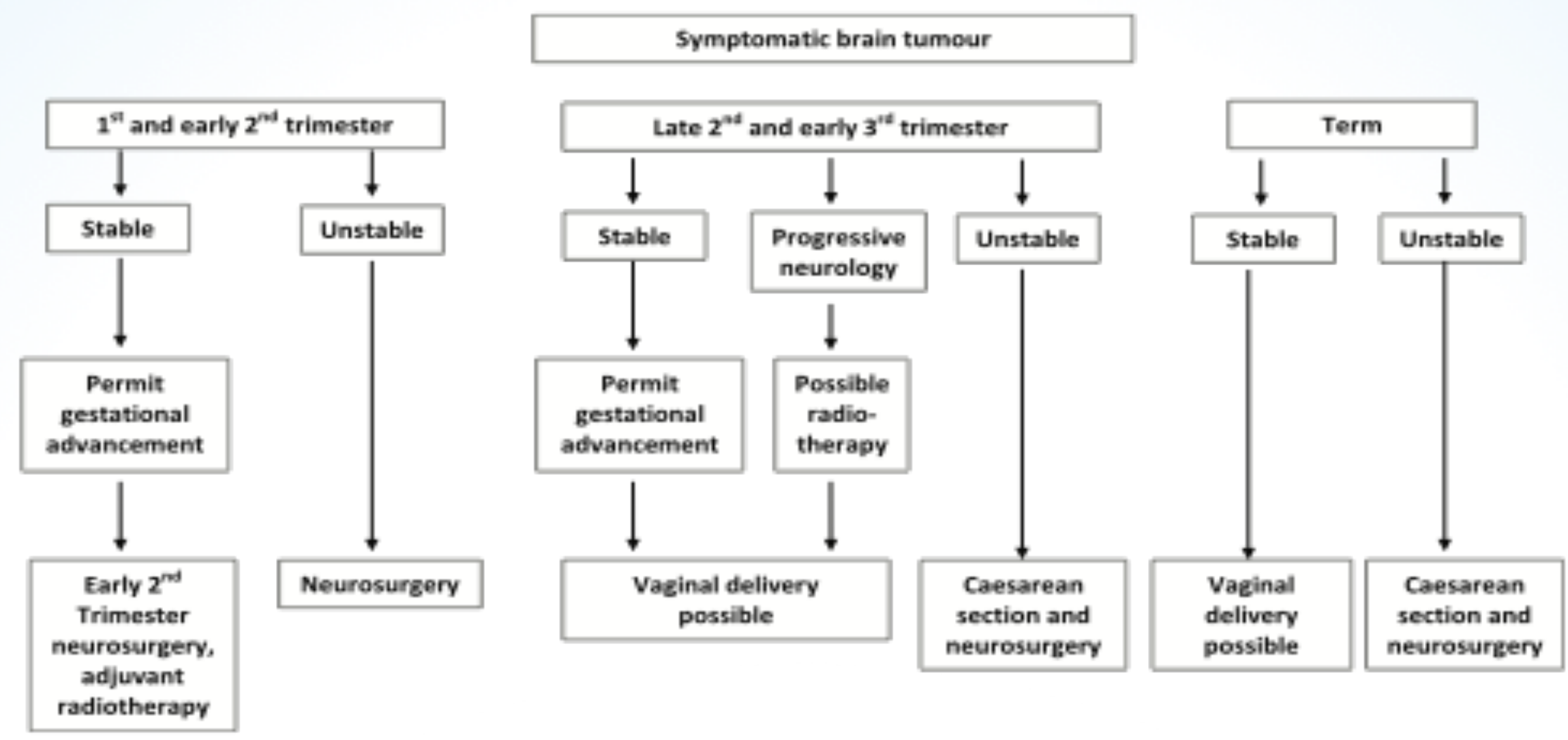

Background: several neurological diseases manifest predominantly in pregnancy. These are usually associated with pregnancy-related conditions: vascular endothelial dysfunction and activation of hormonal receptors on cells surfaces. The aim of this systematic review is to report recent (in the last 10 years) clinical evidence related to anesthetic management of pregnant women undergoing neurosurgery.

Methods: two medical databases Pubmed and Embase, were queried in accordance with Preferred Reporting Items for Systematic Reviews and Metaanalyses (PRISMA) Statement Raccomendations. Key words used for the literature search were: "neuroanesthesia \& pregnancy", "intracranial procedures \& pregnancy", "brain tumor \& pregnancy", "neurosurgery \& pregnancy", "neuroradiology \& pregnancy", "neuroprotection \& pregnancy", "traumatic brain injury \& pregnancy".

Completed studies published in peer-reviewed journals between January 2005 and October 2015 were considered to be eligible: abstract were excluded. The search criteria for inclusion in this review were limited to the following: language (English); study type (human, clinical article, clinical trial, controlled clinical trial, controlled study, randomized controlled trials, case report).

Results: a total of 567 article were retrieved using the listed keywords; 75 were selected and categorized in 3 groups: 1) Brain tumors in pregnancy \& anesthetic management 2) Cerebral vascular lesion in pregnancy (acute subarachnoid hemorrhage in pregnancy and stroke in pregnancy) \& anesthetic management: 3) Traumatic brain injury in pregnancy $\&$ anesthetic management.
Conclusions: anesthetic management involves a multidisciplinary team to ensure the best outcome for mother and fetus. General Anesthesia (GA) seems to be the safest type of anesthesia to administer during neurological procedures in pregnant patients. Cardiotocography (CTG) monitoring seems less useful, as a silent CTG is a normal consequence of general anesthesia. Fetal heart rate monitoring is more accurate, can be performed easily during neurosurgical procedures in pregnant patients; it is sensitive marker to obtain significant information about fetal condition during GA.

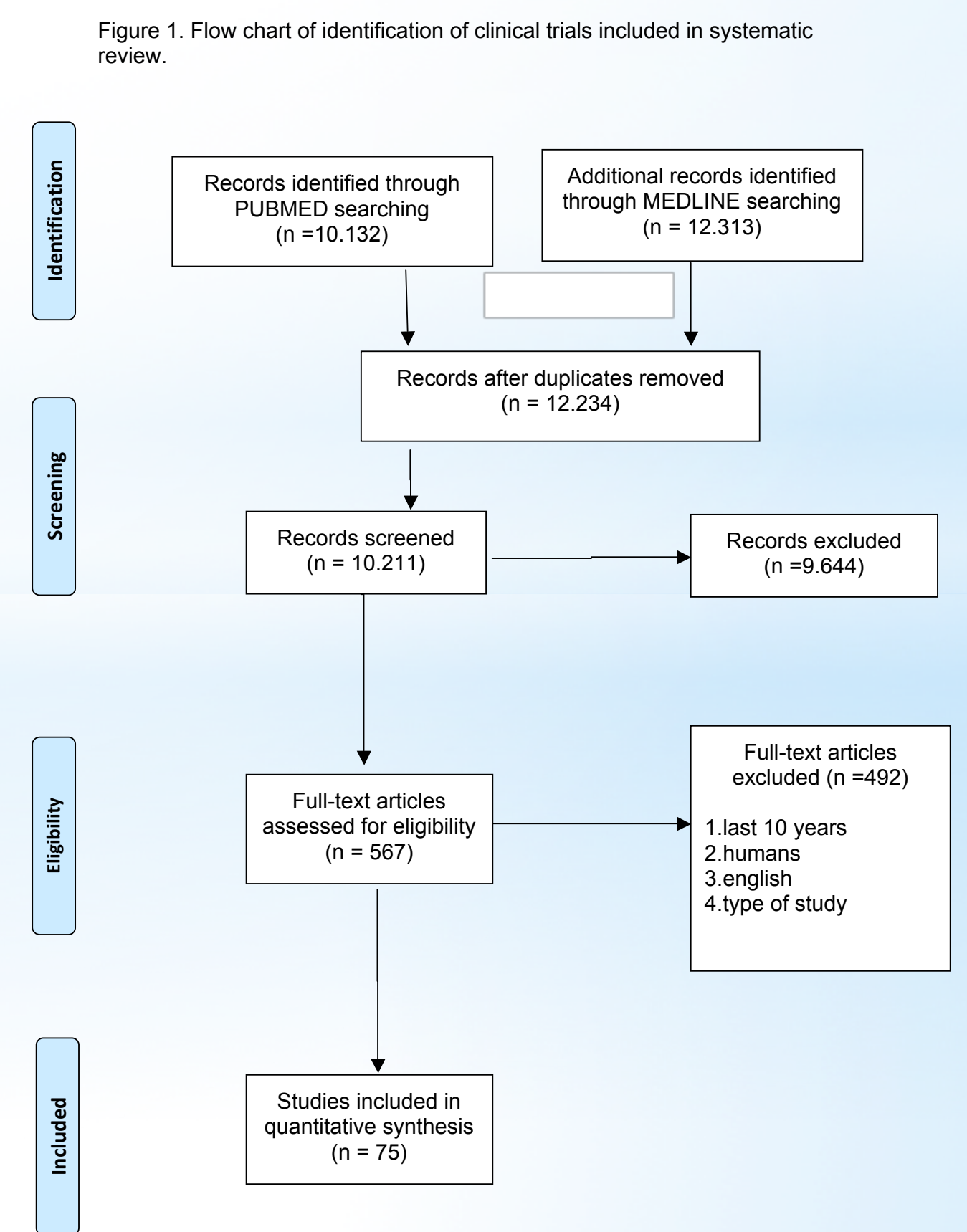

